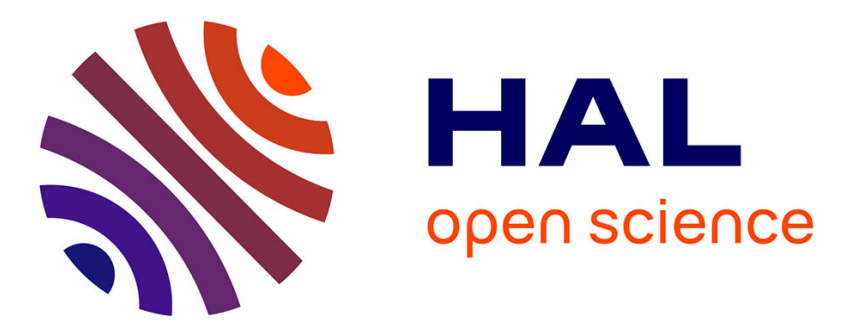

\title{
Passive microrheology of soft materials with atomic force microscopy: A wavelet-based spectral analysis
}

\author{
C. Martinez-Torres, A. Arneodo, L. Streppa, P. Argoul, Françoise Argoul
}

\section{To cite this version:}

C. Martinez-Torres, A. Arneodo, L. Streppa, P. Argoul, Françoise Argoul. Passive microrheology of soft materials with atomic force microscopy: A wavelet-based spectral analysis. Applied Physics Letters, 2016, 108 (3), pp.034102 (1-4). 10.1063/1.4940220 . hal-01275848

\section{HAL Id: hal-01275848 \\ https://hal.science/hal-01275848}

Submitted on 18 Feb 2016

HAL is a multi-disciplinary open access archive for the deposit and dissemination of scientific research documents, whether they are published or not. The documents may come from teaching and research institutions in France or abroad, or from public or private research centers.
L'archive ouverte pluridisciplinaire HAL, est destinée au dépôt et à la diffusion de documents scientifiques de niveau recherche, publiés ou non, émanant des établissements d'enseignement et de recherche français ou étrangers, des laboratoires publics ou privés.

\section{다(1)(2)}

Distributed under a Creative Commons Attribution - ShareAlikel 4.0 International 


\title{
Passive microrheology of soft materials with atomic force microscopy: A wavelet-based spectral analysis
}

\author{
C. Martinez-Torres, ${ }^{1}$ A. Arneodo, ${ }^{1,2}$ L. Streppa, ${ }^{1}$ P. Argoul, ${ }^{3}$ and F. Argoul ${ }^{1,2}$ \\ ${ }^{1}$ CNRS, UMR5672, Laboratoire de Physique, Ecole Normale Supérieure de Lyon, 46 Allée d'Italie, \\ Université de Lyon, 69007 Lyon, France \\ ${ }^{2}$ CNRS, UMR5798, Laboratoire Ondes et Matière d'Aquitaine, Université de Bordeaux, \\ 351 Cours de la Libération, 33405 Talence, France \\ ${ }^{3}$ Université Paris Est, Ecole des Ponts ParisTech, SDOA, MAST, IFSTTAR, 1420 Bd Newton, \\ Cité Descartes, 77420 Champs sur Marne, France
}

\begin{abstract}
Compared to active microrheology where a known force or modulation is periodically imposed to a soft material, passive microrheology relies on the spectral analysis of the spontaneous motion of tracers inherent or external to the material. Passive microrheology studies of soft or living materials with atomic force microscopy (AFM) cantilever tips are rather rare because, in the spectral densities, the rheological response of the materials is hardly distinguishable from other sources of random or periodic perturbations. To circumvent this difficulty, we propose here a wavelet-based decomposition of AFM cantilever tip fluctuations and we show that when applying this multi-scale method to soft polymer layers and to living myoblasts, the structural damping exponents of these soft materials can be retrieved.
\end{abstract}

Local stiffness and internal friction of soft materials (passive or active such as living cells) have lately been addressed at the nanoscale thanks to the development of pico- to nano-Newton force sensing systems and of nanometer resolution position detection devices. ${ }^{1}$ Atomic force microscopy (AFM) is one of these methods, where a sharply tipped flexible cantilever is indented inside a material to extract its local viscoelasticity from the shift and spreading of the cantilever spectral resonance modes. ${ }^{2-4}$ However, these estimations are limited to rather narrow frequency bands surrounding the cantilever resonance modes or their higher harmonics. Spectral decomposition of cantilever fluctuations in contact with soft living tissues in the low frequency range has more rarely been explored. The few attempts which can be found in the literature were performed with small amplitude harmonic excitations $(50 \mathrm{~nm})$ of the sample position driven by a piezo-translator, in the 0.1 to $100 \mathrm{~Hz}$ frequency range, for a small and finite number of frequencies. ${ }^{5,6}$ Whereas passive (driven by thermal fluctuations) microrheology has been performed for the past two decades by a variety of techniques capturing micro-probe spatial fluctuations, ${ }^{7}$ it has not been applied yet to AFM cantilever fluctuations. The limitation of AFM-based passive rheology in the low frequency range comes from the mixing of the background vibrations of the liquid chamber with the cantilever fluctuations given by the rheological response of the material which are difficult to disentangle by standard FFT-based spectral averaging methods. In this work, we show that in quasi-stationary situations, these limitations can be circumvented using a wavelet-based spectral analysis of microcantilever fluctuations under passive excitation. Two experimental applications to passive polymer layers and living adherent myoblast cells are reported.

Based on the generalized Stokes-Einstein relation (GSER) and associated generalizing assumptions, ${ }^{8}$ passive microrheology of soft materials enables the extraction of the frequency-dependent complex modulus $G(\omega)$ which is common to a large class of soft materials (foams, emulsions, slurries, and cells). ${ }^{9-11}$ The observed scaling laws are explained by a characteristic structural disorder and the metastability of these materials which are embodied under the name of "soft glassy materials" or structural damping model. ${ }^{12}$ Their complex shear modulus behaves as

$$
G(\omega)=G_{0}\left(\frac{\omega}{\omega_{0}}\right)^{\alpha}(1+i \eta) \cos \left(\frac{\alpha \pi}{2}\right)
$$

with $\eta=\tan (\alpha \pi / 2)$ as the structural damping coefficient, ${ }^{13}$ $\omega=2 \pi f$ as the radian frequency, and $\alpha$ as a scaling exponent. $G_{0}$ and $\omega_{0}$ are material dependent scaling factors for stiffness and frequency and $G(\omega)=G^{\prime}(\omega)+i G^{\prime \prime}(\omega)$, where $G^{\prime}(\omega)$ is the storage modulus and $G^{\prime \prime}(\omega)$ the loss modulus $\left(i^{2}=-1\right)$. An additional viscous term $i \omega \mu$ can be added to Eq. (1) to include a linear viscous regime at high frequency. Eq. (1) was established from fractional calculus ${ }^{13,14}$ to interpolate between purely elastic $(\alpha=0)$ and purely viscous $(\alpha=1)$ behaviours. When $\alpha$ tends towards 0 , the energy dissipated within a cycle is independent of the frequency ${ }^{15}$ and is proportional to the deformation amplitude $\varepsilon_{m}^{2}$ : $\Delta_{\text {cycle }}$ $=G_{0}\left(\pi^{2} \alpha / 2\right) \varepsilon_{m}^{2}$.

The local deflection signals of an AFM cantilever are produced by different sources of fluctuations. At high frequencies, the hydrodynamic coupling of the cantilever with the surrounding liquid is exciting its resonance modes. At low frequencies, the cantilever captures the acoustic vibrations of the chamber, but also the local random deformations of the material it is in contact with. Each time the material in contact with the cantilever tip breaks locally, or changes its rigidity by fluctuations, the cantilever tip is displaced vertically by $\delta z$. In AFM experiments, the cantilever tip is not 
completely immersed in the viscoelastic medium, so the continuum approach of Stokes equations must be modified to take into account different boundary conditions. The linearized Hertz-Sneddon ${ }^{16}$ equation defines the viscoelastic compliance of the material as proportional to the ratio of the infinitesimal displacement of the tip $\delta z(\omega)=h(\omega)-h_{0}$ inside the media and the cantilever deflection change $\delta d(\omega)$

$$
\chi(\omega)=\frac{1}{G(\omega)}=\frac{8 \tan \theta}{\pi(1-\nu)} \frac{h_{0} \delta z(\omega)}{k_{c} \delta d(\omega)},
$$

where $\theta$ is the half cone tip angle $\left(\sim 15^{\circ}\right), h_{0}$ is the mean depth of indentation of the tip, $\nu$ is the Poisson ratio, and $k_{c}$ is the stiffness constant of the cantilever.

Within the same approximations as those used in GSER, ${ }^{8}$ the spectral density $\mathcal{E}_{z}(\omega)$ of the vertical tip position $z$ can be computed by Fourier transforming its autocovariance function $C_{z}(\tau) \cdot \mathcal{E}_{z}(\omega)$ is related to the imaginary part $\chi^{\prime \prime}(\omega)$ of the medium surrounding the tip ${ }^{17}$

$$
\mathcal{E}_{z}(\omega)=\hat{C}_{z}(\omega)=\int_{\infty}^{\infty} C_{z}(\tau) e^{i \omega \tau} d \tau=-\frac{b k_{B} T \chi^{\prime \prime}(\omega)}{h_{0}},
$$

where $\hat{C}_{z}(\omega)$ denotes the Fourier transform of $C_{z}(\tau)$. $C_{z}(\tau)=\mathbb{E}\{z(t) z(t+\tau)\}$ is even and integrable over $\mathbb{R}$ and decreases fast enough to zero as $\tau \rightarrow \infty$. $\mathbb{E}\{u\}$ is the expectation of $u . h_{0}$ is a characteristic indentation depth depending of the loading force and the visco-elasticity of the sample, $k_{B}$ is the Boltzmann constant and $b=\pi(1-\nu) /(8 \tan \theta)$. When the complex modulus behaves as in Eq. (1), then

$$
\mathcal{E}_{z}(\omega) \propto \omega^{\alpha} .
$$

Thus, the spectral density $\mathcal{E}_{z}(\omega)$ gives access to the imaginary part $\chi^{\prime \prime}(\omega)$ of $\chi(\omega)$ and its real part $\chi^{\prime}(\omega)$ can be obtained by the Kramers-Kronig transformation ${ }^{18}$

$$
\chi^{\prime}(\omega)=\frac{2}{\pi} \mathbb{P} \int_{0}^{\infty} \frac{\zeta \chi^{\prime \prime}(\zeta)}{\zeta^{2}-\omega^{2}} d \zeta
$$

where $\mathbb{P}$ stands for Cauchy principal value. $G(\omega)$ is then obtained by inverting $\chi(\omega)$ (Eq. (2)).

As illustrated in Fig. 1, the spectral density ${ }^{19}$ of the AFM micro-cantilever fluctuations exhibits two different behaviours: at high frequencies, we observe the cantilever resonance modes with a characteristic Lorentzian shape; and at low frequencies (from 150 to $10 \mathrm{kHz}$ in air, and from 10 to $1 \mathrm{kHz}$ in liquid), the spectrum looks similar to white or coloured noise spectra. From the least square fits of the resonance modes, mechanical parameters of the cantilever (stiffness, quality factor, resonance frequency, damping) are usually retrieved. ${ }^{20,21}$ The grey (resp., black) FFT-based spectra plotted in Fig. 1 are computed by FFT transform of the $z$ signals captured at $2.5 \mathrm{MHz}$ rate for $2 \mathrm{~s}\left(5 \times 10^{6}\right.$ points), without averaging (resp. with averaging in log-log scales over frequency windows $\Delta \log _{10} \omega=0.003$ ). It comes out from these two periodograms that without averaging it is impossible to retrieve the shape of the resonance peaks. But let us point out that the averaging is much less efficient at low frequency due to the finite length of the $z$ signal. Outside the resonance regions, these spectra collect a combination of

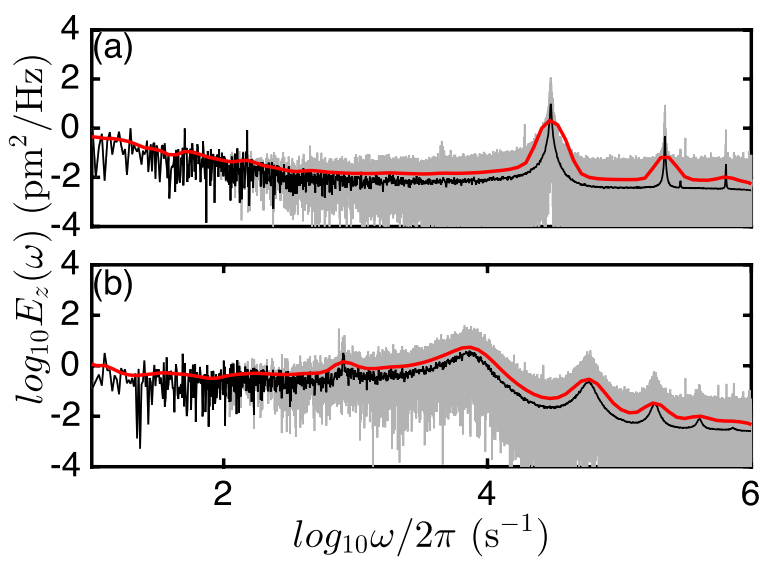

FIG. 1. Comparison of FFT based spectra $\mathcal{E}_{z}(\omega)$ and wavelet based spectra $\mathcal{E}_{z, C W T}(\omega)$ of free cantilever fluctuations in two different media. (a) Air and (b) liquid aqueous chamber. The curves correspond to raw FFT (grey), aver aged FFT (black), and wavelet based (red) spectra.

external sources of noise or vibrations which increase considerably in liquid chambers (Fig. 1(b)) when the cantilever tip is approached to the chamber bottom wall. In the contact limit, the cantilever is used as a local vibration sensor and no more as a vibrating structure with intrinsic resonance modes. ${ }^{20}$

Separating all these sources of fluctuations is a rather tricky problem at low frequencies. ${ }^{22}$ The main drawbacks of FFT-based spectral densities are amplified when scaling laws must be retrieved. Only a sub-interval of the frequencies can actually be used for the linear fit (in a logarithmic representation). The continuous wavelet transform (CWT) is a time-frequency technique that turns out to be very helpful for smoothing the power spectra. ${ }^{19}$ The CWT is defined by a convolution integral proposed in the early eighties by Grossmann et al. ${ }^{23,24}$ using $L^{2}$ normalization

$$
T_{z}(b, a)=\frac{1}{\sqrt{ } a} \int_{\infty}^{\infty} \psi\left(\frac{t-b}{a}\right) z(t) d t .
$$

It can also be written in Fourier space as

$$
T_{z}(b, a)=\frac{\sqrt{ } a}{2 \pi} \int_{\infty}^{\infty} \overline{\hat{\psi}}(a \omega) \hat{z}(\omega) e^{i \omega b} d \omega
$$

$T_{z}(b, a)$ contains information on the signal $z$ at the scale $a$ $=\omega_{\max } / \omega$ around the point $b$ and $\omega_{\max }$ is a reference frequency. ${ }^{25}$ We choose for this study the complex-valued Cauchy analyzing wavelet of order $n:{ }^{15,25,26} \psi(t)=\psi_{n}(t)$ $=(i /(t+i))^{(n+1)}$ with $n=31$. The variance of $T_{z}(b, a)$ can be written as an integral ${ }^{19}$

$$
\begin{aligned}
\mathbb{E}\left\{\left|T_{z}(b, a)\right|^{2}\right\} & =\frac{a}{2 \pi} \int_{\infty}^{\infty} \mathcal{E}_{z}(\omega)|\hat{\psi}(a \omega)|^{2} d \omega, \\
& =\frac{1}{2 \pi} \int_{\infty}^{\infty} \mathcal{E}_{z}\left(\frac{\omega}{a}\right)|\hat{\psi}(\omega)|^{2} d \omega .
\end{aligned}
$$

From the variance of $T_{z}(b, a)$, the wavelet spectral function is defined as

$$
\mathcal{E}_{z, C W T}(a)=\frac{1}{\|\psi\|^{2}} \mathbb{E}\left\{\left|T_{z}(b, a)\right|^{2}\right\} .
$$


For a white noise $z$ of common variance $\sigma^{2}, \mathcal{E}_{z, C W T}(a)=\sigma^{2}$ does not depend on the scale $a$. If the wavelet is chosen such that $\|\psi\|^{2}=1$, the wavelet spectrum function yields an unbiased estimator of spectral power laws. For instance, if $\mathcal{E}_{z}(\omega) \sim \omega^{\beta}$ for $\omega \in \Omega=\left[\omega_{1}, \omega_{2}\right]$, then for a given interval $\left[a_{1}=\omega_{\max } / \omega_{2}, a_{2}=\omega_{\max } / \omega_{1}\right], \mathcal{E}_{z, C W T}(a) \sim a^{\beta}=\left(\omega / \omega_{\max }\right)^{\beta}$. The wavelet spectra of free cantilever fluctuations are plotted in red over the FFT-based spectra (averaged in black, non averaged in grey) in Figs. 1(a) and 1(b) and follow very closely the shape of the cantilever resonance peaks.

The advantage of the wavelet method is to get rid of fluctuations more efficiently than FFT-averaging at low frequencies (because the wavelet averaging window size depends on the scale) and to facilitate the characterization of the out of resonance behaviour when the cantilever comes in contact with a soft material. ${ }^{25}$ This is illustrated in Fig. 2 where we compare $\mathcal{E}_{z}$ and $\mathcal{E}_{z, C W T}$ on five different spectra; the grey line corresponds to a free cantilever in liquid, the black line to the same cantilever tip in hard contact with a glass coverslip, and the blue, green, and red spectra are obtained when the cantilever tip is in contact with a soft polydimethylsiloxane (PDMS) layer. $^{25}$ The noticeable drawback of the wavelet method is the widening of the cantilever resonance modes but their maxima are correctly estimated. The five spectra shown in Fig. 2 reveal some interesting features. When the cantilever is oscillating freely in the liquid chamber (grey curve of Fig. 2(b)), the wavelet spectrum from $f=\omega / 2 \pi=10 \mathrm{~Hz}$ to $f=1 \mathrm{kHz}$ is much lower than when the cantilever is pressed on the bottom coverslip (black curve), and this spectrum changes very little with the frequency $\omega$, reminiscent of an uncorrelated white noise. When the cantilever is placed in contact with the bottom chamber coverslip (black curve), the background vibrations of the liquid chamber are amplified, the largest one emerges around $f$ $=800 \mathrm{~Hz}$. The spectral density of the cantilever fluctuations when it is pressed on a soft layer (PDMS) is drastically different at low frequency $(<8 \mathrm{kHz})$ from that obtained for the same cantilever in contact with a solid coverslip, in particular, the chamber vibration peak is partly damped by the PDMS layer. Its higher frequency resonance modes are

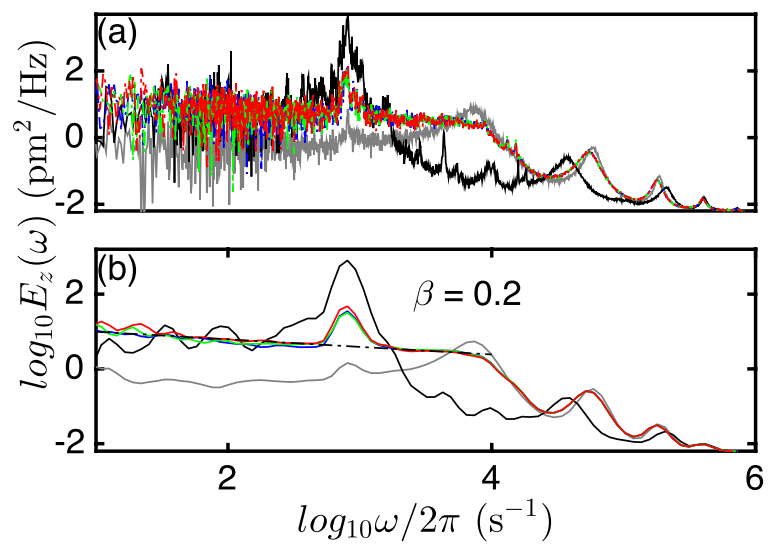

FIG. 2. Comparison of FFT based spectra $\mathcal{E}_{z}(\omega)$ and wavelet spectra $\mathcal{E}_{z, C W T}(\omega)$ of cantilever fluctuations in contact with PDMS thin layers. (a) FFT based spectra. (b) Wavelet based spectra. Grey: free cantilever in liq uid, black: cantilever in hard contact with glass ( $1 \mathrm{nN}$ contact force), blue, (resp., green and red): cantilever in contact with a PDMS thin layer with 0.5 $\mathrm{nN}$ (resp., 1 and $2 \mathrm{nN}$ ) contact force. slightly shifted to lower frequencies (order 2 mode: from $f=59 \mathrm{kHz}$ to $f=53.4 \mathrm{kHz}$ and order 3 mode: from $f$ $=187 \mathrm{kHz}$ to $f=179 \mathrm{kHz}$ ), and its broader resonance peak at $7.3 \mathrm{kHz}$ is flattened in its leftmost part to make place to a flat curve (power-law behaviour). The slope of this line gives an exponent $\beta=0.208 \pm 0.003,{ }^{25}$ the hallmark of a coloured noise signal with persistent correlations. ${ }^{27-29}$ From the slope $\beta$, the exponent $\alpha$ of Eq. (1) can be estimated, in the low frequency limit $(\omega \rightarrow 0)$, as $\alpha=\beta \sim 0.2$. This value of $\alpha$ is characteristic of a viscoelastic material with a more pronounced elasticity than viscosity. Let us point out that this exponent does not change much when increasing the loading force; at this depth of indentation (less than a few micrometers) the material complex modulus is robustly estimated over the range of loading forces considered.

Finally, we have performed the same spectral analysis of the cantilever thermal fluctuations when pressed inside a living myoblast $\left(\mathrm{C} 2 \mathrm{C} 12\right.$ cell). ${ }^{25}$ Fig. 3(a) shows consecutive approach (red)-retract (green) force curves recorded above the nucleus of the adherent myoblast cell shown in Fig. 3(b). We report in Figs. 3(c) and 3(d) the comparison of FFTbased spectra $\mathcal{E}_{z}(\omega)$ with the wavelet-based spectra $\mathcal{E}_{z, C W T}(\omega)$ for a free cantilever in culture media (grey) and for the same cantilever indented inside the cell, above its nucleus, for three loading forces 0.44 (blue), 1.1 (green), and
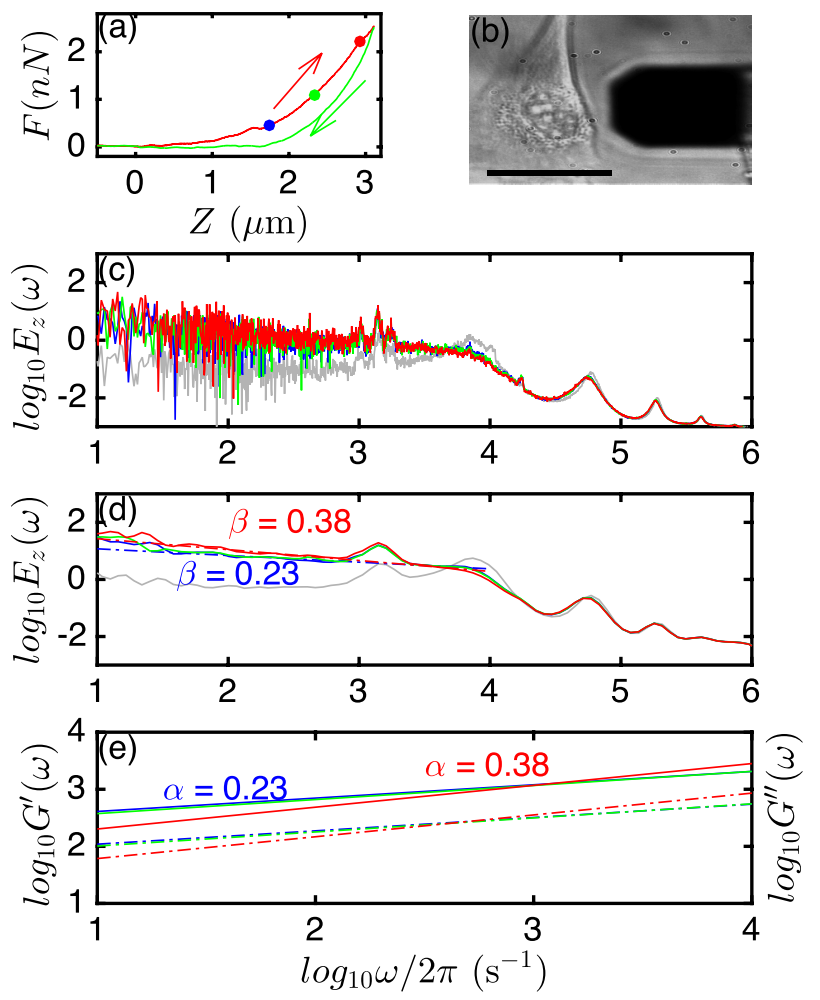

FIG. 3. Comparison of FFT based spectra and wavelet spectra of AFM can tilever fluctuations in pressed within a living myoblast. (a) Approach (red) retract (green) force curves captured on a living C2C12 myoblast cell. (b) Transmission image of the myoblast cell (left) with the cantilever (right) before the scan. Scale bar: $30 \mu \mathrm{m}$. (c) Averaged FFT based spectra. (d) Wavelet based spectra. (e) Corresponding storage $G^{\prime}(\omega)$ (plain lines) and loss $G^{\prime \prime}(\omega)$ (dashed lines) moduli computed with the GSER and Kramers Kronig integral. Grey: free cantilever in liquid, blue: (resp., green and red): cantilever in contact with a $\mathrm{C} 2 \mathrm{C} 12$ myoblast cell with $0.44 \mathrm{nN}$ (resp., 1.1 and $2.2 \mathrm{nN}$ ) loading force. $G^{\prime}(\omega)$ and $G^{\prime \prime}(\omega)$ are expressed in $\mathrm{Pa}$. $\mathcal{E}_{z, C W T}(\omega)$ is given in $\mathrm{pm}^{2} / \mathrm{Hz}$. 
$2.2 \mathrm{nN}$ (red). Similarly to the PDMS layer, we observe a drastic flattening of the spectra, just below the first resonance mode of the cantilever $(f \sim 7 \mathrm{kHz})$ when the cantilever is pressed on the cell. We note also that the FFT-based spectrum of the free cantilever in the culture medium containing motile adherent and floating nonadherent cells (Fig. 3(c)) is much more noisy than the spectrum recorded in pure water (Figs. 1(a) and 2(a)). The presence of cells inside the chamber introduces extra hydrodynamic perturbations that interact with the background vibration modes of the chamber. The wavelet-based spectra are again much smoother (Fig. 3(d)) and the characteristic scaling behavior of structural damping material model emerges again in the lower frequency range $(<6 \mathrm{kHz})$, with a $\beta$ exponent that significantly increases with the loading force. The corresponding $\alpha$ values are 0.23 \pm 0.02 (Ref. 25) (low load) and $0.38 \pm 0.01$ (higher load) as an indicator of some increase of the viscous component when the cantilever is pressed deeper inside the cell, closer to the nucleus. These cells are much softer than the PDMS layer $(G \sim 480 \pm 50 \mathrm{~Pa})$, however, when sensed at low loads, their cytoskeleton (including the cell cortex) keeps a rather high elastic to viscous proportion in their response to stress. For deeper indentations, the increase of $\alpha$ suggests that these deeper zones (related to nucleus responses) are more viscous than the outer parts. It is interesting to note that the three spectra intersect around the frequency $f=1 \mathrm{kHz}$, suggesting that the cantilever fluctuations do not depend on the loading force, as the signature of the ability of the cell to robustly respond to a stress independently of its magnitude. Around $f$ $=1 \mathrm{kHz}$, the energy dissipated inside the cell does not vary with the load.

The values of $\alpha$ estimated at low loads with our passive AFM rheology method are very close to those measured with other techniques with adherent cells ${ }^{11}(\alpha \sim 0.26$ for myoblasts, $\alpha \sim 0.22$ for macrophages, and $\alpha \sim 0.2$ for fibroblasts). It seems that this exponent $\alpha$ would be an invariant of adherent cells, independently of their static shear modulus $G_{0}$. Then, using Eqs. (2) and (5), we can compute the storage $G^{\prime}(\omega)$ and loss $G^{\prime \prime}(\omega)$ moduli of this myoblast cell for the three loading forces (Fig. 3(e)). We note that at frequencies lower that $1 \mathrm{kHz}$, the complex moduli of the cell (real and imaginary parts) are larger for shallow indentation depths (targeting the cytoskeleton) than for deep indentations (contacting the nucleus), whereas at larger frequencies this behaviour is inverted, the deeper indentations giving larger complex moduli. This observation is very interesting since it suggests that the elasticity and viscosity parameters of a cell depend on both the speed at which it is stressed (the higher the frequency, the faster the strain) and the depth of sensing. It also points out the importance of performing spectral studies that would allow us to investigate larger spectral ranges. Our study shows that in the acoustic frequency range (from $10 \mathrm{~Hz}$ to $20 \mathrm{kHz}$ ), the cell behaves as a material with a broad range of delay times and that the distribution of these delays behaves as a power law.

We have shown in this work that AFM can be used for passive rheologic characterization of soft samples (polymers, living cells), thanks to a wavelet-based computation of spectral density of AFM micro-cantilever fluctuations. This method is fast since from a single fluctuation signal, the elastic and viscous characteristics of the sample can be obtained without need of modulating periodically the sample height for a finite set of frequencies. The advantage of AFM, as compared to other particle tracing methods, is to provide some estimate of the low frequency shear modulus $G_{0}$ from force indentation curves and to adapt the range of loading forces to investigate the rheology of soft materials. Furthermore, the possibility to work with cantilevers with different stiffnesses should also allow to further enlarge the frequency range for passive rheology. Indeed, the full performance of this time-frequency decomposition was not used in this work, since we computed a time-averaged variance of the wavelet transform coefficients. To perform a rheological characterization of slowly varying systems, like migrating or dividing cells, a time-frequency decomposition of the complex shear modulus would be very helpful. This work is under progress.

We are very thankful to L. Schaeffer and L. Berguiga for fruitful discussions. This work was supported by the French Agency for Research (ANR-11 IDEX-0007-02 PRES-University of Lyon). The scholarships of C.M.T. and L.S. have been supported, respectively, by CONACyT (Mexico) and AFM-Téléthon.

${ }^{1}$ G. Bao and S. Suresh, Nat. Mater. 2, 715 (2003).

${ }^{2}$ M. Radmacher, R. W. Tillmann, and H. E. Gaub, Biophys. J. 64, 735 (1993).

${ }^{3}$ R. Garcia and R. Perez, Surf. Sci. Rep. 47, 197 (2002).

${ }^{4}$ N. Ploscariu and R. Szoszkiewicz, Appl. Phys. Lett. 103, 263702 (2013).

${ }^{5}$ J. Alcaraz, L. Buscemi, M. Grabulosa, X. Trepat, B. Fabry, R. Farré, and D. Navajas, Biophys. J. 84, 2071 (2003).

${ }^{6}$ P. Roca Cusachs, I. Almendros, R. Sunyer, N. Gavara, R. Farre, and D. Navajas, Biophys. J. 91, 3508 (2006).

${ }^{7}$ T. M. Squires and T. G. Mason, Ann. Rev. Fluid Mech. 42, 413 (2010).

${ }^{8}$ T. G. Mason and D. A. Weitz, Phys. Rev. Lett. 74, 1250 (1995).

${ }^{9}$ F. Gittes, B. Schnurr, P. D. Olmsted, F. C. MacKintosh, and C. F. Schmidt, Phys. Rev. Lett. 79, 3286 (1997).

${ }^{10}$ B. Fabry, G. N. Maksym, J. P. Butler, M. Glogauer, D. Navajas, N. A. Taback, E. J. Millet, and J. J. Fredberg, Phys. Rev. E 68, 041914 (2003).

${ }^{11}$ M. Balland, N. Desprat, D. Icard, S. Féréol, A. Asnacios, J. Browaeys, S. Hénon, and F. Gallet, Phys. Rev. E 74, 021911 (2006).

${ }^{12}$ P. Sollich, Phys. Rev. E 58, 738 (1998).

${ }^{13}$ R. L. Bagley and P. J. Torvik, J. Rheol. 30, 133 (1986).

${ }^{14}$ L. Garibaldi, Viscoelastic Material Damping Technology (Becchis Osiride, Torino, 1996).

${ }^{15} \mathrm{P}$. Argoul, Quelques réflexions sur l'identification de paramètres en dyna mique des matériaux et des structures (HDR, Lyon I University, 2004).

${ }^{16}$ I. N. Sneddon, Int. J. Eng. Sci. 3, 47 (1965).

${ }^{17}$ T. G. Mason, Rheol. Acta 39, 371 (2000).

${ }^{18}$ N. Pottier, Physique Statistique Hors d'Equilibre (CNRS, Paris, 2007).

${ }^{19}$ R. A. Carmona, W. L. Hwang, and B. Torrésani, Practical Time Frequency Analysis (Academic Press, San Diego, 1998).

${ }^{20}$ J. L. Hutter and J. Bechhoefer, Rev. Sci. Instrum. 64, 1868 (1993).

${ }^{21}$ J. W. M. Chon, P. Mulvaney, and J. E. Sader, J. Appl. Phys. 87, 3978 (2000).

${ }^{22}$ T. P. Le and P. Argoul, Mech. Syst. Signal Proc. 52-53, 2945 (2015).

${ }^{23}$ A. Grossmann and J. Morlet, SIAM J. Math. Anal. 15, 723 (1984).

${ }^{24} \mathrm{~J}$. M. Combes, A. Grossmann, and P. Tchamitchian, Wavelets. Time Frequency Methods and Phase Space (Springer Verlag, Berlin, 1990).

${ }^{25}$ See supplementary material at http://dx.doi.org/10.1063/1.4940220 for a more detailed description of materials and methods, polymer layers prepa ration, cell culture, and wavelet transform analysis.

${ }^{26}$ P. Argoul and T. P. Le, Mech. Syst. Signal Proc. 17, 243 (2003).

${ }^{27}$ J. F. Muzy, E. Bacry, and A. Arneodo, Int. J. Bifurcation Chaos 4, 245 (1994).

${ }^{28}$ A. Arneodo, E. Bacry, and J. F. Muzy, Physica A 213, 232 (1995).

${ }^{29}$ B. Audit, E. Bacry, J. F. Muzy, and A. Arneodo, IEEE Trans. Inf. Theory 48, 2938 (2002). 